\title{
Psychiatric complications (depression, anxiety disorders, post-traumatic stress disorder) of ARDS: a systematic review
}

Nela Klimcik

CHU Dijon: Centre Hospitalier Universitaire de Dijon

Maxime Nguyen

CHU Dijon: Centre Hospitalier Universitaire de Dijon

Philippe D'Athis

CHU Dijon: Centre Hospitalier Universitaire de Dijon

Nathalie Forestier

CHU Dijon: Centre Hospitalier Universitaire de Dijon

Pierre-grégoire Guinot

CHU Dijon: Centre Hospitalier Universitaire de Dijon

Jean Christophe Chauvet Gelinier

CHU Dijon: Centre Hospitalier Universitaire de Dijon

Belaid Bouhemad ( $\nabla$ belaid_bouhemad@hotmail.com )

CHU Dijon: Centre Hospitalier Universitaire de Dijon https://orcid.org/0000-0002-6124-2156

\section{Research Article}

Keywords: acute respiratory distress syndrome, depression, anxiety disorders, post-traumatic stress disorder, prevalence, risk factor

Posted Date: June 15th, 2021

DOI: https://doi.org/10.21203/rs.3.rs-514080/v1

License: (a) (1) This work is licensed under a Creative Commons Attribution 4.0 International License. Read Full License 


\section{Abstract}

Background: ARSDS requires extensive resuscitation and has major physical sequelae. We evaluated the psychiatric sequelae by conducting a systematic review to determine prevalence of symptoms of depression, anxiety disorders and post-traumatic stress disorder (PTSD) following ARDS, as well as their risk factors.

Methods: We conducted a search on Pubmed by including all studies meeting our primary endpoint (prevalence of symptoms of depression, anxiety disorders and PTSD following ARDS) and secondary endpoint (associated risk factors).

Results: Included studies found prevalence ranging from 16 to $46 \%$ for symptoms of depression, 23 to $62 \%$ for symptoms of anxiety disorders and 16 to $47 \%$ for symptoms of PTSD. The data were too heterogeneous to conduct a meta-analysis.

Among the risk factors associated with a risk factor of depression, anxiety disorders or PTSD after ARDS were; factors related to patient (as an history of mental illness) risk factors related to ICU management (as use opioids and benzodiazepine). Effect of severity of ARDS is not known.

Conclusion: This systematic review shows that depression, anxiety disorders and PTSD following ARDS are frequent, long-lasting, disabling and have severe psychiatric consequences. Associated risk factors can be identified and among us use of opioids.

\section{Introduction}

It is known that there can be psychiatric consequences following a stay in ICU [1] and it seems likely that ARDS, which requires long and invasive management in ICU, can also be the cause of significant psychiatric comorbidities, of equal or greater frequency, especially as many of the physical sequelae of ARDS can be disabling in the long term. The link between depression and chronic pathologies has also been demonstrated.[2] Although many studies have evaluated the prevalence of co-morbidities following ARDS, there is to date no recent systematic review of the psychiatric complications (depression, anxiety disorders, post-traumatic stress disorder (PTSD)) of ARDS. However, a better understanding of the psychiatric sequelae of these patients would be valuable for psychiatrists, whether in current practice or in a context where we are regularly affected by new infectious diseases that generate ARDS, such as SARS ten years ago or COVID-19, more recently. [3]

The objective of this systematic review of all the studies in populations of ARDS survivors in the Medline database corresponding to the inclusion factors, is to have data on the prevalence and risk factors for symptoms of depression, anxiety disorders and PTSD following the management of ARDS in resuscitation.

\section{Methods}




\section{Eligibility criteria}

We searched for studies assessing the prevalence of the symptomatology of depression and/or anxiety disorders and/or PTSD following ARDS management. They could be longitudinal studies, prospective cohort studies, retrospective case-control studies. Randomized controlled trials that looked for the influence of a therapeutic parameter or component on depression and/or anxiety disorders and/or PTSD were also included if they looked for the prevalence of depression and/or anxiety disorders and/or PTSD in their population.

The following exclusion criteria were applied: case studies, commentaries on articles or editorials, studies looking for the validity of a scale, symptomatology of depression and/or anxiety disorders, and/or PTSD not assessed by specific scales and validated or diagnosed by a psychiatrist, and studies of less than thirty patients, in order to exclude studies of insufficient statistical power. However, the presence of a control group was not essential for a study to be included in the review.

\section{Outcome}

The analysis of the primary endpoint involved compiling the prevalence of depression (and/or) anxiety disorders (and/or) PTSD in the included studies. In addition to the main analysis, a subgroup analysis was performed for studies involving patient populations that required ECMO-VV management.

The secondary endpoint analysis consisted of collecting risk factors significantly associated in multivariate analysis with the risk of depression (and/or) anxiety disorders (and/or) PTSD. To be included in our analysis, the association or its absence had to be expressed as a numerical value $(\beta$, relative risk or odds ratio) accompanied by a confidence interval.

\section{Search strategy}

A literature search was conducted using the following electronic databases: Scopus, PubMed, Embase, CINAHL, Web of Science, and the Cochrane Library. The search was done according to the following formula (acute respiratory distress syndrome OR acute lung injury) AND (depression OR anxiety OR posttraumatic stress disorder). The search was conducted without any article format, data, nor language restrictions, and included studies published until April 15, 2020. Other references were also identified through reading previous systematic reviews and in reading the bibliographies of the included studies

\section{Study selection and bias risk assessment}

The titles for the articles retrieved from the search were manually reviewed by two authors. Only abstracts of original investigations were included and we removed letters to the editor, reviews, abstracts only, and non-article formats. All abstracts were read by two people, and if the studies met the inclusion and exclusion criteria after this reading, they were included in the final analysis and read in their entirety in order to extract from them the primary and secondary judgment criteria. The references of all included articles and those from selected reviews were checked for relevancy. The following data were also 
extracted: year of publication, country in which the study was conducted, period of conduct of the study, inclusion and exclusion criteria, details on interventions, and characteristics of the studies.

If there was disagreement between the two readers of the abstracts, a third person decided whether or not to include the study in the journal. The quality of the included studies was assessed using the validated STROBE grid and the results of the qualitative analysis were synthesized in tabular form and subjected to meta-analysis for the main judgement criterion.

\section{Results}

\section{Selected studies}

The search initially returned 382 studies, 30 studies met the inclusion and exclusion criteria and were therefore read in full. Of these, 23 were selected for the final qualitative analysis [4-26]. The process of study selection is shown using a flow chart. (Fig.1)

The studies included in the review therefore looked for either the prevalence of symptoms of depression, the prevalence of symptoms of anxiety disorders, the prevalence of symptoms of PTSD, or the prevalence of symptoms of several of these conditions in the aftermath of ARDS. The main features of the 23 studies included in the review are summarized in Table 1.

\section{Prevalence of Depressive Symptoms}

We included 17 studies in the systematic review looking for the prevalence of symptoms of depression following ARDS in their population $(n=3713)$. Data are summarized in Table 2. In qualitative analysis, a prevalence range of 16 to $46 \%$ was found across all studies and $25 \%$ to $39 \%$ in ECMO patients (Table 2 ).

Unfortunately, the great heterogeneity of the data (highly different durations of observation to assess prevalence) did not allow to carry out the meta-analysis originally planned in the design of our review.

\section{Prevalence of Anxiety Disorder Symptoms}

We included 13 studies and the systematic review and looked for the prevalence of symptoms of anxiety disorders following ARDS in their population $(n=3497)$. The data are summarized in Table 3 . In qualitative analysis, a range of prevalence from 23 to $62 \%$ was found across all studies and $34 \%$ to $40 \%$ in ECMO patients (Table 3).

\section{Prevalence of symptoms of post-traumatic stress disorder}

We included 12 studies in the systematic review looking for the prevalence of PTSD symptoms following ARDS in their population ( $n=3817)$. The data are summarized in Table 3. In qualitative analysis, a prevalence ranging from 16 to $47 \%$ was found across all studies. The prevalence was $16 \%$ to $47 \%$ in ECMO patients (Table 3 ). 


\section{Co-occurrences of symptoms of depression, anxiety disorders and PTSD}

Only 3 studies in our review examined the co-occurrences of the 3 mental illnesses studied and reported the following results: co-occurrence of symptoms of 2 mental illnesses fom 20 to 60\%.[16, 19, 20]; cooccurrence of symptoms of 3 mental illnesses from 20 to 40\%.[16, 19] A co-occurrence of symptoms of depression and anxiety disorders in $18 \%$ of patients, a co-occurrence of symptoms of anxiety disorders and PTSD in $22 \%$ of patients, and a co-occurrence of symptoms of depression and PTSD in $3 \%$ of patients were reported.[20]

\section{Risk factors significantly associated in multivariate analysis with the risk of depression, anxiety disorders or PTSD}

The risk factors associated with symptoms of depression, anxiety disorders and PTSD are presented respectively in Tables 5, 6 and 7. All associations found in multivariate analysis are expressed with the confidence interval found in the studies of the systematic review.

Risk factors can be divided in 3 categories.

- Patient-dependent risk factors: past history of mental illness, female gender, young age, low socio educational level unemployment or disability, high BMI and presence of co-morbidities or a history of addictions.

- Risk factors associated with severity of the disease.

-Risk factors associated with used drugs or ICU stay: In particular, high opioid consumption was found associated with symptoms of depression, anxiety disorders and PTSD.

\section{Discussion}

For the symptoms of the 3 psychiatric conditions in the aftermath of ARDS the systematic review found prevalence ranging between $16-46 \%$ for depression, $23-62 \%$ for anxiety disorders and $16-47 \%$ for PTSD, with comparable results in various collection periods. Furthermore, some risk factors associated with the development of these psychiatric conditions following ARDS, related to ICU management (use of opioids and benzodiazepines), can be highlighted.

Most studies assess the presence or absence of psychiatric symptoms using psychometric scales, but not a clinical diagnosis by a psychiatrist. Therefore our study referred to the symptoms of a given pathology and not to the pathology itself. Indeed, studies with diagnosis made by psychiatrists are difficult to conduct. We included only studies using validated scales in the diagnosis of depression, anxiety disorders and PTSD. The used scales were all validated by the literature as being sensitive and specific for the diagnoses, whether it is the HADS [27, 28] Arnau, 2001 \#28\}, the BDI[29], the Zung SelfRating Depression Scale [30], the BAI [31, 32], the IES-R[33], the IES [34], the PTSS-10 [35]. This suggests that the results synthesized in our review are well predictive of the diagnoses of depression, anxiety 
disorders and PTSD. Unfortunately, the insufficient and too heterogeneous data of our study did not allow a meta-analysis which could have allowed us to obtain a figure more representative of reality. Globally, the prevalence of depression worldwide is estimated at $6 \%$ and it is estimated that $15 \%-18 \%$ of the world population will be affected by depression at least once in their lifetime [36]; the population of ARDS survivors therefore appears to be at greater risk than the general population developing depressive disorder [36]. Similarly, the estimated global prevalence of anxiety disorders ranges worldwide ranges from $3.8 \%$ to $25 \%$ [37]) and the prevalence of PTSD has been estimated at 3.9\%. [38] Our results are therefore higher than those for the general population, but are comparable to those found in studies of populations that have been managed in intensive care units. [1,39]

For risk factors associated by multivariate analysis with the development of depression, anxiety disorders and PTSD following ARDS, the data in the current state of research (especially those from studies with a correct methodology and expressed with a confidence interval), are still scarce (in studies of psychiatric disorders following either ARDS or, more generally, resuscitation management) and do not allow any real conclusions to be drawn. However, some slight trends should be highlighted:

People with a history of mental illness are at greater risk of developing after ARDS depression, anxiety disorders or PTSD. Certain factors associated with the patient such as female gender, young age (for anxiety disorders), low socio educational level (for depression), unemployment or disability, high BMI (for both depression and anxiety disorders), co-morbidities (for anxiety disorders) or a history of addictions also appear to induce greater risk of developing mental illness. Indeed, it is now accepted that a history of depression, anxiety disorder or post-traumatic stress disorder makes the patient more at risk of developing a mental disorder again, as it is known that mental pathologies are often associated with each other. Moreover young age, female gender, socio-economic difficulties, chronic pathologies and addictive pathologies are also risk factors for depression, anxiety disorders and PTSD. [40]

Risk factors associated with intensive care management seem to be generally weakly associated with psychiatric symptoms following ARDS. Only heavy opioid use was associated with the risk of symptoms of the 3 psychiatric pathologies studied, and heavy benzodiazepine use was found for 2 pathologies as a risk factor associated with depression in multivariate analysis.

The severity of initial impairment presents contradictory results, because it was found once as a risk factor for anxiety disorders and PTSD, and also found once as a protective factor against depression and PTSD. Finally, hypoglycemia was found once to be a risk factor for depression (with a strong level of evidence because of the association established by a randomized trial) and once as a risk factor for PTSD, and high corticosteroid consumption was found once to be a protective factor against anxiety disorders and once against PTSD. Returning to work seems to protect against depression, and cognitive disorders seem to favor anxiety disorders (associations established by one of the studies in the review).

Our study is the first systematic review[41] on the prevalence of psychiatric symptoms (depression, anxiety disorders, PTSD) including studies conducted since the reduction of mortality rate of ARDS (which fell from $50 \%$ in the 1990 s to $33 \%$ in the 2000s).[42] And survivors are more likely to develop 
somatic and psychiatric complications during the convalescence phase, which is one of the challenges of ARDS management.[43-45] However, very few studies involving ECMO-VV patients were available to perform analysis in management of ARDS, but the prevalence found is similar to that found in other populations managed for ARDS.

\section{Limitations}

This systematic review has some limitations. Firstly, the heterogeneity of the studies included, due to their methodological differences (a few retrospective studies with a lower level of evidence were included with mostly prospective studies), but also due to the heterogeneous measurement instruments, made impossible a meta-analysis. Secondly, diagnostic scales tend to overestimate the prevalence of the pathologies as they detect pathologies with high sensitivity and low specificity.[23, 46] Moreover, the semi-structured psychiatric interview remains the reference to diagnose mental illness combining the exhaustive aspect of a scale and the clinical finesse of a face-to-face interview with an experienced practitioner.[47] In our review, only 2 studies of PTSD used the SCID-II, one as first-line and one as diagnostic confirmation after a scale. $[5,12]$ Thirdly, the inclusion criteria for studies are also sometimes heterogeneous, some excluding patients with a history of mental illness and others not, considering mental illness only as a risk factor. In addition, many studies have reported difficulties to determine some of the patients' characteristics prior to their ARDS, particularly with regard to psychiatric history. Fourthly, we have tried to synthesize at best the associations found in different studies, which used different ways to define certain variables. In particular, different thresholds were used for numerical variables such as age, biological values or the consumption of certain drugs. It would have been too complex to take this into account, so we have described each association generically without numerical values. Finally, since we only included studies published until April 15, 2020, we did not include very recent publications.[48]

\section{Conclusion}

Beyond the long-term physical consequences of ARDS, our review shows that there are also frequent, long-lasting, disabling and severe psychiatric consequences. While much research is still needed, it seems important for physicians, and particularly psychiatrists, to better understand and detect the symptoms of depression, anxiety disorders and PTSD, and thus to continue the work carried out in intensive care, to improve the health and quality of life of patients and their families.

\section{Declarations}

-Ethical Approval and Consent to participate: Not applicable

-Consent for publication: Not applicable

-Availability of supporting data: Not applicable

-Competing interests: All authors listed on the title page have no competing interest to declare 
-Funding: Solely on departmental fund

\section{-Authors' contributions:}

NK: this author contributed to the study concept, performed the data collection and drafted the manuscript.

MN: this author contributed to the study concept and design; performed the data analysis, and drafted the manuscript.

PA: this author performed the data analysis, and substantially revised the manuscript.

NF: this author contributed to the study concept and performed the data collection.

PGG: this author contributed to the study concept, the analysis of data, and substantially revised the manuscript.

JCCG: this author contributed to the study concept and substantially revised the manuscript.

BB: this author contributed to the study concept and design, performed the data analysis and drafted the manuscript.

\section{All authors read and approved the final manuscript}

Acknowledgements: none

\section{References}

1. Hatch R, Young D, Barber V, Griffiths J, Harrison DA, Watkinson P, (2018) Anxiety, Depression and Post Traumatic Stress Disorder after critical illness: a UK-wide prospective cohort study. Crit Care 22: 310

2. Read JR, Sharpe L, Modini M, Dear BF, (2017) Multimorbidity and depression: A systematic review and meta-analysis. J Affect Disord 221: 36-46

3. Park M, Cook AR, Lim JT, Sun Y, Dickens BL, (2020) A Systematic Review of COVID-19 Epidemiology Based on Current Evidence. J Clin Med 9

4. Deja M, Denke C, Weber-Carstens S, Schroder J, Pille CE, Hokema F, Falke KJ, Kaisers U, (2006) Social support during intensive care unit stay might improve mental impairment and consequently healthrelated quality of life in survivors of severe acute respiratory distress syndrome. Crit Care 10: R147

5. Schelling G, Stoll C, Haller M, Briegel J, Manert W, Hummel T, Lenhart A, Heyduck M, Polasek J, Meier M, Preuss U, Bullinger M, Schuffel W, Peter K, (1998) Health-related quality of life and posttraumatic stress disorder in survivors of the acute respiratory distress syndrome. Crit Care Med 26: 651-659 
6. Orme J, Jr., Romney JS, Hopkins RO, Pope D, Chan KJ, Thomsen G, Crapo RO, Weaver LK, (2003) Pulmonary function and health-related quality of life in survivors of acute respiratory distress syndrome. Am J Respir Crit Care Med 167: 690-694

7. Hopkins RO, Weaver LK, Chan KJ, Orme JF, Jr., (2004) Quality of life, emotional, and cognitive function following acute respiratory distress syndrome. J Int Neuropsychol Soc 10: 1005-1017

8. Hopkins RO, Weaver LK, Collingridge D, Parkinson RB, Chan KJ, Orme JF, Jr., (2005) Two-year cognitive, emotional, and quality-of-life outcomes in acute respiratory distress syndrome. Am J Respir Crit Care Med 171: 340-347

9. Dowdy DW, Dinglas V, Mendez-Tellez PA, Bienvenu OJ, Sevransky J, Dennison CR, Shanholtz C, Needham DM, (2008) Intensive care unit hypoglycemia predicts depression during early recovery from acute lung injury. Crit Care Med 36: 2726-2733

10. Dowdy DW, Bienvenu OJ, Dinglas VD, Mendez-Tellez PA, Sevransky J, Shanholtz C, Needham DM, (2009) Are intensive care factors associated with depressive symptoms 6 months after acute lung injury? Crit Care Med 37: 1702-1707

11. Adhikari NKJ, McAndrews MP, Tansey CM, Matte A, Pinto R, Cheung AM, Diaz-Granados N, Barr A, Herridge MS, (2009) Self-reported symptoms of depression and memory dysfunction in survivors of ARDS. Chest 135: 678-687

12. Hauer D, Weis F, Krauseneck T, Vogeser M, Schelling G, Roozendaal B, (2009) Traumatic memories, post-traumatic stress disorder and serum cortisol levels in long-term survivors of the acute respiratory distress syndrome. Brain Res 1293: 114-120

13. Hopkins RO, Key CW, Suchyta MR, Weaver LK, Orme JF, Jr., (2010) Risk factors for depression and anxiety in survivors of acute respiratory distress syndrome. Gen Hosp Psychiatry 32: 147-155

14. Adhikari NKJ, Tansey CM, McAndrews MP, Matte A, Pinto R, Cheung AM, Diaz-Granados N, Herridge MS, (2011) Self-reported depressive symptoms and memory complaints in survivors five years after ARDS. Chest 140: 1484-1493

15. Bienvenu OJ, Colantuoni E, Mendez-Tellez PA, Dinglas VD, Shanholtz C, Husain N, Dennison CR, Herridge MS, Pronovost PJ, Needham DM, (2012) Depressive symptoms and impaired physical function after acute lung injury: a 2-year longitudinal study. Am J Respir Crit Care Med 185: 517-524

16. Mikkelsen ME, Christie JD, Lanken PN, Biester RC, Thompson BT, Bellamy SL, Localio AR, Demissie E, Hopkins RO, Angus DC, (2012) The adult respiratory distress syndrome cognitive outcomes study: long-term neuropsychological function in survivors of acute lung injury. Am J Respir Crit Care Med 185: $1307-1315$ 
17. Stevenson JE, Colantuoni E, Bienvenu OJ, Sricharoenchai T, Wozniak A, Shanholtz C, MendezTellez PA, Needham DM, (2013) General anxiety symptoms after acute lung injury: predictors and correlates. J Psychosom Res 75: 287-293

18. Bienvenu OJ, Gellar J, Althouse BM, Colantuoni E, Sricharoenchai T, Mendez-Tellez PA, Shanholtz C, Dennison CR, Pronovost PJ, Needham DM, (2013) Post-traumatic stress disorder symptoms after acute lung injury: a 2-year prospective longitudinal study. Psychol Med 43: 2657-2671

19. Bienvenu OJ, Colantuoni E, Mendez-Tellez PA, Shanholtz C, Dennison-Himmelfarb CR, Pronovost PJ, Needham DM, (2015) Cooccurrence of and remission from general anxiety, depression, and posttraumatic stress disorder symptoms after acute lung injury: a 2-year longitudinal study. Crit Care Med 43: $642-653$

20. Huang M, Parker AM, Bienvenu OJ, Dinglas VD, Colantuoni E, Hopkins RO, Needham DM, National Institutes of Health NHL, Blood Institute Acute Respiratory Distress Syndrome N, (2016) Psychiatric Symptoms in Acute Respiratory Distress Syndrome Survivors: A 1-Year National Multicenter Study. Crit Care Med 44: 954-965

21. Spencer-Segal JL, Hyzy RC, Iwashyna TJ, Standiford TJ, (2017) Psychiatric Symptoms in Survivors of Acute Respiratory Distress Syndrome. Effects of Age, Sex, and Immune Modulation. Ann Am Thorac Soc 14: 960-967

22. Bienvenu OJ, Friedman LA, Colantuoni E, Dinglas VD, Sepulveda KA, Mendez-Tellez P, Shanholz C, Pronovost PJ, Needham DM, (2018) Psychiatric symptoms after acute respiratory distress syndrome: a 5-year longitudinal study. Intensive Care Med 44: 38-47

23. Kapfhammer HP, Rothenhausler HB, Krauseneck T, Stoll C, Schelling G, (2004) Posttraumatic stress disorder and health-related quality of life in long-term survivors of acute respiratory distress syndrome. Am J Psychiatry 161: 45-52

24. Mikkelsen ME, Shull WH, Biester RC, Taichman DB, Lynch S, Demissie E, Hansen-Flaschen J, Christie JD, (2009) Cognitive, mood and quality of life impairments in a select population of ARDS survivors. Respirology 14: 76-82

25. Schmidt M, Zogheib E, Roze H, Repesse X, Lebreton G, Luyt CE, Trouillet JL, Brechot N, Nieszkowska A, Dupont H, Ouattara A, Leprince P, Chastre J, Combes A, (2013) The PRESERVE mortality risk score and analysis of long-term outcomes after extracorporeal membrane oxygenation for severe acute respiratory distress syndrome. Intensive Care Med 39: 1704-1713

26. Sanfilippo F, Ippolito M, Santonocito C, Martucci G, Carollo T, Bertani A, Vitulo P, Pilato M, Panarello G, Giarratano A, Arcadipane A, (2019) Long-term functional and psychological recovery in a population of acute respiratory distress syndrome patients treated with VV-ECMO and in their caregivers. Minerva Anestesiol 85: 971-980 
27. Bjelland I, Dahl AA, Haug TT, Neckelmann D, (2002) The validity of the Hospital Anxiety and Depression Scale. An updated literature review. J Psychosom Res 52: 69-77

28. Jutte JE, Needham DM, Pfoh ER, Bienvenu OJ, (2015) Psychometric evaluation of the Hospital Anxiety and Depression Scale 3 months after acute lung injury. J Crit Care 30: 793-798

29. Arnau RC, Meagher MW, Norris MP, Bramson R, (2001) Psychometric evaluation of the Beck Depression Inventory-II with primary care medical patients. Health Psychol 20: 112-119

30. Thurber S, Snow M, Honts CR, (2002) The Zung Self-Rating Depression Scale: convergent validity and diagnostic discrimination. Assessment 9: 401-405

31. Beck AT, Epstein N, Brown G, Steer RA, (1988) An inventory for measuring clinical anxiety: psychometric properties. J Consult Clin Psychol 56: 893-897

32. Geissner E, Huetteroth A, (2018) [Beck Anxiety Inventory German Version - A Reliable, Valid, Patientfriendly Instrument for Measuring Clinical Anxiety]. Psychother Psychosom Med Psychol 68: 118125

33. Hosey MM, Bienvenu OJ, Dinglas VD, Turnbull AE, Parker AM, Hopkins RO, Neufeld KJ, Needham DM, (2019) The IES-R remains a core outcome measure for PTSD in critical illness survivorship research. Crit Care 23: 362

34. Sundin EC, Horowitz MJ, (2002) Impact of Event Scale: psychometric properties. Br J Psychiatry 180: $205-209$

35. Rosendahl J, Kisyova H, Gawlytta R, Scherag A, (2019) Comparative validation of three screening instruments for posttraumatic stress disorder after intensive care. J Crit Care 53: 149-154

36. Malhi GS, Mann J, (2018) Depression. Lancet 392: 2299-2312.

37. Remes O, Brayne C, van der Linde R, Lafortune L, (2016) A systematic review of reviews on the prevalence of anxiety disorders in adult populations. Brain Behav 6: e00497

38. Koenen KC, Ratanatharathorn A, Ng L, McLaughlin KA, Bromet EJ, Stein DJ, Karam EG, Meron Ruscio A, Benjet C, Scott K, Atwoli L, Petukhova M, Lim CCW, Aguilar-Gaxiola S, Al-Hamzawi A, Alonso J, Bunting B, Ciutan M, de Girolamo G, Degenhardt L, Gureje O, Haro JM, Huang Y, Kawakami N, Lee S, Navarro-Mateu F, Pennell BE, Piazza M, Sampson N, Ten Have M, Torres Y, Viana MC, Williams D, Xavier M, Kessler RC, (2017) Posttraumatic stress disorder in the World Mental Health Surveys. Psychol Med 47: 2260-2274

39. Griffiths J, Fortune G, Barber V, Young JD, (2007) The prevalence of post traumatic stress disorder in survivors of ICU treatment: a systematic review. Intensive Care Med 33: 1506-1518 
40. Tortella-Feliu M, Fullana MA, Perez-Vigil A, Torres X, Chamorro J, Littarelli SA, Solanes A, Ramella-Cravaro V, Vilar A, Gonzalez-Parra JA, Andero R, Reichenberg A, Mataix-Cols D, Vieta E, Fusar-Poli P, loannidis JPA, Stein MB, Radua J, Fernandez de la Cruz L, (2019) Risk factors for posttraumatic stress disorder: An umbrella review of systematic reviews and meta-analyses. Neurosci Biobehav Rev 107: 154165

41. Davydow DS, Desai SV, Needham DM, Bienvenu OJ, (2008) Psychiatric morbidity in survivors of the acute respiratory distress syndrome: a systematic review. Psychosom Med 70: 512-519

42. Chiumello D, Coppola S, Froio S, Gotti M, (2016) What's Next After ARDS: Long-Term Outcomes. Respir Care 61: 689-699

43. Herridge MS, Moss M, Hough CL, Hopkins RO, Rice TW, Bienvenu OJ, Azoulay E, (2016) Recovery and outcomes after the acute respiratory distress syndrome (ARDS) in patients and their family caregivers. Intensive Care Med 42: 725-738

44. Iwashyna TJ, (2010) Survivorship will be the defining challenge of critical care in the 21st century. Ann Intern Med 153: 204-205

45. Davydow DS, Zatzick DF, (2009) Surviving critical illness is not without its perils: a perspective on depression in acute lung injury survivors. Crit Care Med 37: 1817-1818

46. Stoll C, Kapfhammer HP, Rothenhausler HB, Haller M, Briegel J, Schmidt M, Krauseneck T, Durst K, Schelling G, (1999) Sensitivity and specificity of a screening test to document traumatic experiences and to diagnose post-traumatic stress disorder in ARDS patients after intensive care treatment. Intensive Care Med 25: 697-704

47. Pettersson A, Bostrom KB, Gustavsson P, Ekselius L, (2015) Which instruments to support diagnosis of depression have sufficient accuracy? A systematic review. Nord J Psychiatry 69: 497-508

48. Mongodi S, Salve G, Tavazzi G, Politi P, Mojoli F, team C-P-I, Unit C-PC, (2021) High prevalence of acute stress disorder and persisting symptoms in ICU survivors after COVID-19. Intensive Care Med

\section{Tables}

Table 1 Presentation of studies included in the systematic review after reading the full articles 


\section{Prospective interventional studies}

Deja et al. 2006 [4] randomized

1 hospital

1991 - 2000 Median time: 57

controlled months

\section{Prospective observational cohort} studies

Schelling et al., $\quad$ Prospective

1998 [5]

Orme et al., 2003 [6]

Hopkins et

al.,2004 [7]

Hopkins et al.,

2005 [8]

Dowdy et al.,

2008, [9]

Dowdy et al.

2009 [10]

Adhikari et al.,

2009, [11]

Hauer et al.,

2009 [12]

Hopkins et al.,

2010 [13]

Adhikari et al.,

2011 [14]

Bienvenu et al.

,2012, [15]

Mikkelsen et al.,

2012, [16]

Stevenson et al.,

2013, [17]

Bienvenu et al.

2013 [18]

Bienvenu et et al.,

2015, [19]

Huang et al.

2016, [20]

cohort

cohort

cohort

cohort

cohort

cohort,

cohort

cohort

cohort

cohort

cohort

cohort

cohort

cohort

cohort

cohort
1 ICU from 1 hospital

1 ICU from 1 hospital

Prospective

Prospective

Prospective

Prospective

Prospective 13 ICU in 4 hospitals

Prospective ICU from 4 hospitals

Prospective Data base population.

Prospective a previous ARDS study

Prospective ICU from 4 hospitals

Prospective 13 ICU in 4 hospitals

Prospective ICUs from28 hospitals

Prospective 13 ICU in 4 hospitals

Prospective 13 ICU in 4 hospitals

Prospective

Prospective

ICU in 4 hospitals
1985 - 1995 Median time: 4 years

$1994-19991$ year

1994 - 19991 year

1994 - 19992 years

$2004-2007 \quad 3$ months

$2004-2007 \quad 6$ months

$1998-2001 \quad 1$ year

Unspecified Variable

$1994-1999 \quad 1$ and 2 years

$1998-2001 \quad 5$ years

$2004-2007 \quad 3$ and 6 months, 1 and 2 years

$2000-20051$ year

$2004-20073$ months

$2004-2007 \quad 3$ and 6 months, 1 and

2 years

$2004-2007 \quad 2$ years

41 ARDS Network hospitals No defined

Collection at 6 months and 1 year 
Spencer-Segal et al. 2017, [21]

Bienvenu et al., 2018 [22]
Prospective cohort

Prospective 13 ICU in 4 hospitals cohort
6 ICU from 3 hospitals
$2004-20096$ months

$2004-2007$

3 and 6 months, 1,2 and 5 years

\section{Retrospective studies}

\begin{tabular}{|c|c|c|c|c|}
\hline $\begin{array}{l}\text { Kapfhammer et al., } \\
2004 \text { [23] }\end{array}$ & $\begin{array}{l}\text { Retrospective } \\
\text { cohort }\end{array}$ & $\begin{array}{l}\text { data collection from } 1 \\
\text { hospital }\end{array}$ & $1985-1995$ & Median time: 8 years \\
\hline $\begin{array}{l}\text { Mikkelsen et } \\
\text { al.,2009 [24] }\end{array}$ & Retrospective & 1 ARDS survivor support site & No defined & Variable collection \\
\hline $\begin{array}{l}\text { Schmidt et al. } \\
2013 \text { [25] }\end{array}$ & Retrospective & $\begin{array}{l}\text { Patients treated with ECMO- } \\
\text { VV in } 3 \text { ICU }\end{array}$ & $2008-2012$ & 6 months \\
\hline $\begin{array}{l}\text { Sanfilippo et al. } \\
\text { 2019, [26] }\end{array}$ & Retrospective & $\begin{array}{l}\text { patients treated with ECMO- } \\
\text { VV from ICU }\end{array}$ & $2009-2016$ & $\begin{array}{l}\text { Collection in } 2017 \text { for } \\
\text { all patients }\end{array}$ \\
\hline
\end{tabular}

Table 2 Prevalence of Depressive Symptoms by Study 


\begin{tabular}{|c|c|c|c|c|c|c|c|c|c|}
\hline \multirow[t]{2}{*}{ Study } & \multirow{2}{*}{$\begin{array}{l}\text { Scale } \\
\text { used }\end{array}$} & \multirow{2}{*}{$\begin{array}{c}\text { Population } \\
\text { characteristics }\end{array}$} & \multirow{2}{*}{$\begin{array}{c}\text { Number of } \\
\text { patients } \\
\text { analyzed at the } \\
\text { end* }\end{array}$} & \multicolumn{6}{|c|}{ Time of examination } \\
\hline & & & & $\begin{array}{r}3 \\
\text { months }\end{array}$ & $\begin{array}{r}6 \\
\text { months }\end{array}$ & $\begin{array}{r}1 \\
\text { year }\end{array}$ & $\begin{array}{l}2 \\
\text { years }\end{array}$ & 5 years & $\begin{array}{c}\text { Not } \\
\text { defined }\end{array}$ \\
\hline $\begin{array}{l}\text { Dowdy, } \\
2008 \text { [9] }\end{array}$ & $\begin{array}{l}\text { HAD- } \\
D>8\end{array}$ & $\begin{array}{l}\text { Mean age } 48 \text { years, } \\
\text { F/M ratio }(46 \% / 54 \%)\end{array}$ & 104 & $28 \%$ & & & & & \\
\hline $\begin{array}{l}\text { Dowdy, } \\
2009 \text { [10] }\end{array}$ & $\begin{array}{l}\mathrm{HAD}- \\
\mathrm{D} \geq 8\end{array}$ & $\begin{array}{l}\text { Mean age } 49 \text { years, } \\
\text { F/M ratio }(44 \% / 56 \%)\end{array}$ & 160 & & $26 \%$ & & & & \\
\hline $\begin{array}{l}\text { Bienvenu, } \\
\text { 2012[15] }\end{array}$ & $\begin{array}{l}\text { HAD- } \\
\mathrm{D} \geq 8\end{array}$ & $\begin{array}{l}\text { Mean age } 49 \text { years, } \\
\text { F/M ratio }(44 \% / 56 \%)\end{array}$ & $\begin{array}{c}174 \text { (3 months) } \\
174(6 \\
\text { months) } \\
156(1 \text { year }) \\
146 \text { (2 years) }\end{array}$ & $26 \%$ & $25 \%$ & $24 \%$ & $32 \%$ & & \\
\hline $\begin{array}{l}\text { Schmidt, } \\
2013 \text { [25] }\end{array}$ & $\begin{array}{l}\text { HAD- } \\
\mathrm{D} \geq 8\end{array}$ & $\begin{array}{l}\text { Mean age } 37 \text { years, } \\
\text { F/M ratio }(54 \% / 46 \%)\end{array}$ & 67 & & $25 \%$ & & & & \\
\hline $\begin{array}{l}\text { Bienvenu, } \\
2015 \text { [19] }\end{array}$ & $\begin{array}{l}\mathrm{HAD}- \\
\mathrm{D} \geq 8\end{array}$ & $\begin{array}{l}\text { Mean age } 49 \text { years, } \\
\text { F/M ratio }(44 \% / 56 \%)\end{array}$ & $\begin{array}{c}174 \text { (3 months) } \\
173 \text { (6 months) } \\
156 \text { (1year) } \\
146 \text { ( } 2 \text { years })\end{array}$ & $26 \%$ & & & $33 \%$ & & $46 \%{ }^{\dagger}$ \\
\hline $\begin{array}{l}\text { Huang, } \\
2016[20]\end{array}$ & $\begin{array}{l}\text { HAD- } \\
\text { D }>8\end{array}$ & $\begin{array}{l}\text { Mean age } 49 \text { years, } \\
\text { F/M ratio }(52 \% / 48 \%)\end{array}$ & $\begin{array}{c}613 \text { (6 months) } \\
576 \text { (12 } \\
\text { months) }\end{array}$ & & $36 \%$ & $36 \%$ & & & \\
\hline $\begin{array}{l}\text { Spencer- } \\
\text { Segal, } \\
2017 \text { [21] }\end{array}$ & $\begin{array}{l}\text { HAD- } \\
\text { D>8 }\end{array}$ & $\begin{array}{l}\text { Placebo: Mean age } 49 \text {, } \\
\text { F/M ratio } \\
\text { (36.9\%/63.1\%); GM- } \\
\text { CSF: Mean age } 50 \text { F/M } \\
\text { ratio }(48 \% / 52 \%)\end{array}$ & 132 & & $32 \% \$$ & & & & \\
\hline $\begin{array}{l}\text { Bienvenu, } \\
2018 \text { [22] }\end{array}$ & $\begin{array}{l}\text { HAD- } \\
\mathrm{D} \geq 8\end{array}$ & $\begin{array}{l}\text { Mean age } 49 \text { years, } \\
\text { F/M ratio }(44 \% / 56 \%)\end{array}$ & $\begin{array}{c}\text { At } 3 \text { and } 6 \\
\text { months, } 1 \text { and } 2 \\
\text { years see } \\
\text { [19] } 123 \text { (5 } \\
\text { years) }\end{array}$ & & & & & & $32 \% \square$ \\
\hline $\begin{array}{l}\text { San } \\
\text { Filippo, } \\
2019 \text { [26] }\end{array}$ & $\begin{array}{l}\text { HAD- } \\
\text { D }>7\end{array}$ & $\begin{array}{l}\text { Mean age } 41.3, \mathrm{~F} / \mathrm{M} \\
\text { ratio }(27 \% / 73 \%)\end{array}$ & 33 & & & & & & $39 \%$ \\
\hline $\begin{array}{l}\text { Adhikari, } \\
2009 \text { [11] }\end{array}$ & $\begin{array}{l}\text { BDI- } \\
\mathrm{II} \geq 20\end{array}$ & $\begin{array}{l}\text { Mean age } 42 \text { years, } \\
\text { F/M ratio }(46 \% / 54 \%)\end{array}$ & 61 & & & & & & $41 \%$ \\
\hline $\begin{array}{l}\text { Adhikari, } \\
2011 \text { [14] }\end{array}$ & $\begin{array}{l}\text { BDI- } \\
\mathrm{II} \geq 20\end{array}$ & $\begin{array}{l}\text { Median age } 48 \text { years, } \\
\text { F/M ratio }(48 \% / 52 \%)\end{array}$ & 43 & & & & & $18,60 \%$ & \\
\hline $\begin{array}{l}\text { Orme, } \\
2003[6]\end{array}$ & $\begin{array}{l}\mathrm{BDI}> \\
16\end{array}$ & $\begin{array}{l}\text { Mean age } 46 \text { years, } \\
\text { F/M ratio }(55 \% / 45 \%)\end{array}$ & 66 & & & $16 \%$ & & & \\
\hline $\begin{array}{l}\text { Hopkins, } \\
2004 \text { [7] }\end{array}$ & $\begin{array}{l}\text { BDI> } \\
16\end{array}$ & $\begin{array}{l}\text { Mean age } 46 \text { years, } \\
\text { F/M ratio ( } 55 \% / 45 \%)\end{array}$ & 66 & & & $16 \%$ & & & \\
\hline $\begin{array}{l}\text { Hopkins, } \\
2005[8]\end{array}$ & $\begin{array}{l}\mathrm{BDI}> \\
16\end{array}$ & $\begin{array}{l}\text { Mean age } 46 \text { years, } \\
\text { F/M ratio }(55 \% / 45 \%)\end{array}$ & 62 & & & & $23 \%$ & & \\
\hline $\begin{array}{l}\text { Hopkins, } \\
2010[13]\end{array}$ & $\begin{array}{l}\mathrm{BDI}> \\
16\end{array}$ & $\begin{array}{l}\text { Mean age } 46 \text { years old, } \\
\text { F/M ratio }(55 \% / 45 \%)\end{array}$ & $\begin{array}{l}66 \text { ( } 1 \text { year }) \\
62 \text { (2 years) }\end{array}$ & & & $16 \%$ & $23 \%$ & & \\
\hline
\end{tabular}

HAD-D: Hospital Anxiety and Depression Scale; GM-CSF: granulocyte macrophage-colony stimulating factor; BDI: Beck Depression Inventory, F/M ration (female/male ratio); *: number of patients actually assessed for depressive symptoms according to the study protocol, not including the deceased and those lost to follow-up. ${ }^{\dagger}$ : of the total population, to at least one 
collection; ${ }^{\text {: }}$ prolonged depressive symptomatology over 5 years; $\$$ : valid prevalence for the entire study population

Table 3 Prevalence of anxiety disorder symptoms by studies 


\begin{tabular}{|c|c|c|c|c|c|c|c|c|}
\hline \multirow[t]{2}{*}{ Study } & \multirow{2}{*}{$\begin{array}{l}\text { Uses } \\
\text { scale }\end{array}$} & \multirow[t]{2}{*}{ Population characteristics } & \multirow{2}{*}{$\begin{array}{l}\text { Number of } \\
\text { patients } \\
\text { analyzed at } \\
\text { the end* }\end{array}$} & \multicolumn{4}{|c|}{ Time of examination } & \multirow{2}{*}{$\begin{array}{c}\text { Not } \\
\text { defined }\end{array}$} \\
\hline & & & & $\begin{array}{c}3 \\
\text { months }\end{array}$ & $\begin{array}{c}6 \\
\text { months }\end{array}$ & 1 year & $\begin{array}{c}2 \\
\text { years }\end{array}$ & \\
\hline $\begin{array}{c}\text { Stevenson, } \\
2013 \text { [17] }\end{array}$ & $\mathrm{HAD}-\mathrm{A}>8$ & $\begin{array}{c}\text { Median age } 49 . \mathrm{F} / \mathrm{M} \text { ratio } \\
(41 \% / 59 \%)\end{array}$ & 152 & $38 \%$ & & & & \\
\hline $\begin{array}{l}\text { Bienvenu, } \\
\text { 2015, [19] }\end{array}$ & $\mathrm{HAD}-\mathrm{A}>8$ & $\begin{array}{l}\text { Average age } 49 \text { years, } \mathrm{F} / \mathrm{M} \\
\text { ratio }(44 \% / 56 \%)\end{array}$ & $\begin{array}{c}174(3 \\
\text { months }) \\
173(6 \\
\text { months }) \\
156 \text { (1year) } \\
146 \text { (2 years) }\end{array}$ & $38 \%$ & & & $44 \%$ & ${ }_{56 \%} \dagger$ \\
\hline $\begin{array}{l}\text { Bienvenu, } \\
2018 \text { [22] }\end{array}$ & $\mathrm{HAD}-\mathrm{A}>8$ & $\begin{array}{l}\text { Average age } 49 \text { years, } \mathrm{F} / \mathrm{M} \\
\text { ratio }(44 \% / 56 \%)\end{array}$ & $\begin{array}{l}\text { At } 3 \text { and } 6 \\
\text { months, } 1 \text { and } \\
2 \text { years }[19] \\
123 \text { (5 years) }\end{array}$ & & & & & $52 \%^{\square}$ \\
\hline $\begin{array}{l}\text { Orme, } \\
2003[6]\end{array}$ & $\mathrm{BAI}>16$ & $\begin{array}{c}\text { Average age } 46 \text { years, } \mathrm{F} / \mathrm{M} \\
\text { ratio }(55 \% / 45 \%)\end{array}$ & 66 & & & $24 \%$ & & \\
\hline $\begin{array}{l}\text { Hopkins, } \\
2004 \text { [7] }\end{array}$ & $\mathrm{BAI}>16$ & $\begin{array}{l}\text { Average age } 46 \text { years, } \mathrm{F} / \mathrm{M} \\
\text { ratio }(55 \% / 45 \%)\end{array}$ & 66 & & & $24 \%$ & & \\
\hline $\begin{array}{l}\text { Hopkins, } \\
2005[8]\end{array}$ & $\mathrm{BAI}>16$ & $\begin{array}{c}\text { Average age } 46 \text { years, } \mathrm{F} / \mathrm{M} \\
\text { ratio }(55 \% / 45 \%)\end{array}$ & 62 & & & & $23 \%$ & \\
\hline \multirow{2}{*}{$\begin{array}{l}\text { Hopkins, } \\
2010 \text { [13] }\end{array}$} & \multirow[t]{2}{*}{$\mathrm{BAI}>16$} & \multirow{2}{*}{$\begin{array}{l}\text { Average age } 46 \text { years, } \mathrm{F} / \mathrm{M} \\
\quad \text { ratio }(55 \% / 45 \%)\end{array}$} & 66 (1 year) & & & $24 \%$ & $23 \%$ & \\
\hline & & & 62 (2 years) & & & & & \\
\hline $\begin{array}{l}\text { Mikkelsen, } \\
2009[24]\end{array}$ & $\begin{array}{c}\text { BAI } \\
\text { (threshold } \\
\text { not } \\
\text { specified) }\end{array}$ & $\begin{array}{c}\text { Average age } 43.3, \mathrm{~F} / \mathrm{M} \text { ratio } \\
(84 \% / 16 \%)\end{array}$ & 79 & & & & & $48 \%$ \\
\hline $\begin{array}{l}\text { Mikkelsen, } \\
2012 \text { [16] }\end{array}$ & $\begin{array}{c}\text { BAI } \\
\text { (threshold } \\
\text { not } \\
\text { specified) }\end{array}$ & $\begin{array}{c}\text { Average age } 49 \text { years, } \mathrm{F} / \mathrm{M} \\
\text { ratio }(57 \% / 43 \%)\end{array}$ & 102 & & & $62 \%$ & & \\
\hline $\begin{array}{l}\text { Sanfilippo, } \\
2019 \text { [26] }\end{array}$ & $\mathrm{HAD}-\mathrm{A}>7$ & $\begin{array}{c}\text { Average age } 41.3, \mathrm{~F} / \mathrm{M} \text { ratio } \\
(27 \% / 73 \%)\end{array}$ & 33 & & & & & $40 \%$ \\
\hline $\begin{array}{l}\text { Schmidt, } \\
2013 \text { [25] }\end{array}$ & $\mathrm{HAD}-\mathrm{A}>8$ & $\begin{array}{c}\text { Average age } 37 \text { years, } \mathrm{F} / \mathrm{M} \\
\text { ratio }(54 \% / 46 \%)\end{array}$ & 67 & & $34 \%$ & & & \\
\hline $\begin{array}{l}\text { Huang, } \\
2016[20]\end{array}$ & $\mathrm{HAD}-\mathrm{A}>8$ & $\begin{array}{c}\text { Average age } 49 \text { years, } \mathrm{F} / \mathrm{M} \\
\text { ratio }(52 \% / 48 \%)\end{array}$ & $\begin{array}{l}613(6 \\
\text { months }) \\
576(12 \\
\text { months })\end{array}$ & & $42 \%$ & $42 \%$ & & \\
\hline $\begin{array}{l}\text { Schmidt, } \\
2013[25]\end{array}$ & $\mathrm{HAD}-\mathrm{A}>8$ & $\begin{array}{c}\text { Average age } 37 \text { years, } \mathrm{F} / \mathrm{M} \\
\text { ratio ( } 54 \% / 46 \%)\end{array}$ & 67 & & $34 \%$ & & & \\
\hline $\begin{array}{l}\text { Spencer- } \\
\text { Segal, } \\
2017[21]\end{array}$ & $\mathrm{HAD}-\mathrm{A}>8$ & $\begin{array}{c}\text { Placebo: Mean age } 49, \mathrm{~F} / \mathrm{M} \\
\text { ratio }(37 \% / 63 \%) ; \mathrm{GM}-\mathrm{CSF} \text { : } \\
\text { Mean age } 50, \mathrm{~F} / \mathrm{M} \text { ratio } \\
(48 \% / 52 \%)\end{array}$ & 132 & & $32 \% \boldsymbol{\$}$ & & & \\
\hline
\end{tabular}

HAD-A: Hospital Anxiety and Depression Scale (part of the scale about anxiety disorders); BAI: Beck Anxiety Inventory; GM-CSF: granulocyte macrophage-colony stimulating factor; 
BDI: Beck Depression Inventory, F/M ration (female/male ratio); *: number of patients actually assessed for symptoms of anxiety disorders according to the study protocol, not including the deceased and those lost to follow-up; ${ }^{\dagger}$ : out of the general population, at least one caseload; ${ }^{\mathrm{a}}$ : percentage of patients with symptoms of prolonged anxiety disorders over the five years; $\$$ : prevalence valid for the entire study population;

Table 4 Prevalence of PTSD symptoms by studies 


\begin{tabular}{|c|c|c|c|c|c|c|c|c|}
\hline \multirow[t]{2}{*}{ Study } & \multirow[t]{2}{*}{ Scales } & \multirow{2}{*}{$\begin{array}{c}\text { Population } \\
\text { characteristics }\end{array}$} & \multirow{2}{*}{$\begin{array}{c}\text { Number of } \\
\text { patients } \\
\text { analyzed at the } \\
\text { end* }\end{array}$} & \multicolumn{4}{|c|}{ Time of examination } & \multirow[b]{2}{*}{$\begin{array}{c}\text { Not } \\
\text { defined }\end{array}$} \\
\hline & & & & $\begin{array}{c}3 \\
\text { months }\end{array}$ & 6 months & 1 year & $\begin{array}{c}2 \\
\text { years }\end{array}$ & \\
\hline $\begin{array}{l}\text { Bienvenu, } \\
2013 \text { [18] }\end{array}$ & IES-R $\geq 1.6$ & $\begin{array}{l}\text { Mean age } 49 \\
\text { years, } \\
\text { F/M ratio } \\
(44 \% / 56 \%)\end{array}$ & $\begin{array}{c}174 \text { (3 months) } \\
173 \text { (6 months) } \\
156 \text { (1year) } \\
146 \text { ( } 2 \text { years }\end{array}$ & $24 \%$ & $20 \%$ & $23 \%$ & $24 \%$ & \\
\hline $\begin{array}{l}\text { Bienvenu, } \\
\text { 2015, [19] }\end{array}$ & IES-R $\geq 1.6$ & $\begin{array}{c}\text { Mean age } 49 \\
\text { years, } \\
\text { F/M ratio } \\
(44 \% / 56 \%)\end{array}$ & $\begin{array}{l}3 \text { months }(174) \\
6 \text { months }(173) \\
12 \text { months }(156) \\
24 \text { months (146) }\end{array}$ & & $22 \%$ & & $24 \%$ & $33 \%{ }^{\dagger}$ \\
\hline $\begin{array}{l}\text { Huang, } \\
2016 \text { [20] }\end{array}$ & IES-R $>1.6$ & $\begin{array}{c}\text { Mean age } 49 \\
\text { years, } \\
\text { F/M ratio } \\
(52 \% / 48 \%)\end{array}$ & $\begin{array}{c}613 \text { (6months) } \\
576 \text { (1 year) }\end{array}$ & & $24 \%$ & $23 \%$ & & \\
\hline $\begin{array}{l}\text { Bienvenu, } \\
2018 \text { [22] }\end{array}$ & IES-R $\geq 1.6$ & $\begin{array}{c}\text { Mean age } 49 \\
\text { years, } \\
\text { F/M ratio } \\
(44 \% / 56 \%)\end{array}$ & $\begin{array}{c}\text { At } 3 \text { and } 6 \\
\text { months, } 1 \text { and } 2 \\
\text { years [19] } \\
123 \text { (5 years) }\end{array}$ & & & & & $23 \%^{\square}$ \\
\hline $\begin{array}{c}\text { Sanfilippo, } \\
2019 \text { [26] }\end{array}$ & $\begin{array}{l}\text { IES-R (cut-off } \\
21 \text { and 33) }\end{array}$ & $\begin{array}{c}\text { Mean age } 41 \\
\text { years, } \\
\text { F/M ratio } \\
(27 \% / 73 \%)\end{array}$ & 33 & & & & & $\begin{array}{c}47 \% \\
\text { and } 21 \\
\% \text { (cut- } \\
\text { off } 21 \\
\text { and } \\
33 \text { ) }\end{array}$ \\
\hline $\begin{array}{l}\text { Schmidt, } \\
2013 \text { [25] }\end{array}$ & IES $\geq 30$ & $\begin{array}{c}\text { Mean age } 37 \\
\text { years, } \\
\text { F/M ratio } \\
(54 \% / 46 \%)\end{array}$ & 67 & & $16 \%$ & & & \\
\hline $\begin{array}{c}\text { Schelling, } \\
1998 \text { [5] }\end{array}$ & PTSS-10 >35 & $\begin{array}{l}\text { Non-ECMO: } \\
\text { Mean age } 36 \\
\text { years } \\
\text { F/M ratio } \\
\text { (51\%/49\%) } \\
\text { ECMO: } \\
\text { Mean age } 34 \\
\text { years } \\
\text { F/M ratio } \\
(35 \% / 65 \%)\end{array}$ & $\begin{array}{c}80 \\
\text { Non-ECMO : } 66 \\
\text { ECMO :14 }\end{array}$ & & & & & $28 \% \$$ \\
\hline $\begin{array}{c}\text { Deja, } 2006 \\
{[4]}\end{array}$ & PTSS-10 >35 & $\begin{array}{c}\text { Mean age } 33 \\
\text { years, } \\
\text { F/M ratio } \\
(40 \% / 60 \%)\end{array}$ & 65 & & & & & $29 \%$ \\
\hline $\begin{array}{l}\text { Hauer, } \\
2009 \text { [12] }\end{array}$ & $\begin{array}{l}\text { PTSS-10 and } \\
\text { confirmation } \\
\text { with SCID-II }\end{array}$ & $\begin{array}{c}\text { Mean age } 40 \\
\text { years } \\
\text { F/M ratio } \\
(52 \% / 48 \%)\end{array}$ & 33 & & & & & $29 \%$ \\
\hline $\begin{array}{c}\text { Mikkelsen, } \\
2012 \text { [16] }\end{array}$ & PTSS-10>35 & $\begin{array}{c}\text { Mean age } 49 \\
\text { years, } \\
\text { F/M ratio } \\
(57 \% / 43 \%)\end{array}$ & 102 & & & $39 \%$ & & \\
\hline $\begin{array}{l}\text { Spencer- } \\
\text { Segal, }\end{array}$ & $\begin{array}{c}\text { PTSS-10 }>35 \text { ) } \\
\text { and IES }>19\end{array}$ & $\begin{array}{l}\text { Placebo: } \\
\text { Mean age } 49 \\
\text { years }\end{array}$ & 132 & & $\begin{array}{c}20 \% \\
\text { (PTSS- }\end{array}$ & & & \\
\hline
\end{tabular}


GM-CSF:

Mean age 50

years

$\mathrm{F} / \mathrm{M}$ ratio

(48\%/52\%)

Kapfhammer,

Interviews via

Mean age 36.5

46

$44 \%$

2004 [23]

SCID by 2

years

psychiatrists

$\mathrm{F} / \mathrm{M}$ ratio

(48\%/52.\%)

Clinical Interview for the DSM-IV; F/M ratio (Female/Male ratio); *i.e., number of patients actually assessed for symptoms of PTSD according to the study protocol, not including the

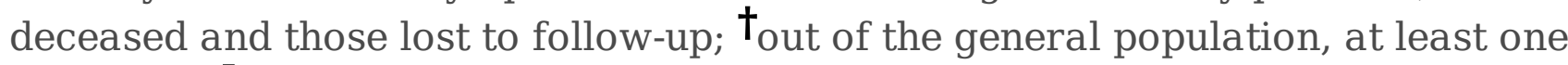
collection; : percentage of patients with symptoms of prolonged PTSD over five years; $\$$ prevalence valid for the entire study population 
Table 5 Factor associated with depression in ARDS patients

\begin{tabular}{|c|c|}
\hline Potential Risk & Risk factors for ARDS \\
\hline
\end{tabular}

\begin{tabular}{|c|c|c|}
\hline Age & & $\begin{array}{l}\underline{\mathbf{1 5}} \mathrm{OR}=1.13(0.93-1.36)(\mathrm{B}) ; \underline{\mathbf{1 8}} 1.03(0.95 . \\
1.12) ;(\mathrm{B}) \underline{\mathbf{2 2}} \text { OR } 0.6(0.3-1.2)(\mathrm{B}) ; \underline{\mathbf{2 1}} \beta=-0.058 \\
(-0.14 .0 .028) \text { (B) }\end{array}$ \\
\hline Women & $\underline{20} \mathrm{RR}=1.26$ (1.01. 1.58); (B) & $\begin{array}{l}\underline{\mathbf{1 5}} \mathrm{OR}=0.70(0.39-1.24(\mathrm{~B}) ; \underline{\mathbf{2 2}} \text { OR } 1.7(0.9-3.2) \\
(\mathrm{B}) ; \underline{\mathbf{2 1}} \beta=-0.15(-2.6 .2 .3)(\mathrm{B})\end{array}$ \\
\hline High BMI & $\begin{array}{l}\underline{\mathbf{1 0}} 1.9(1.0 .3 .4)(\mathrm{B}) ; \underline{\mathbf{9}} \mathrm{RR}=3.3 \\
(1.2 .4 .2)(\mathrm{B})\end{array}$ & $\begin{array}{l}\underline{\mathbf{1 5}} \mathrm{OR}=1.05(0.56-1.96)(\mathrm{B}) ; \underline{\mathbf{2 0}} \mathrm{RR}=1.07(0.96 . \\
1.20)(\mathrm{B})\end{array}$ \\
\hline $\begin{array}{l}\text { Unemployment } \\
\text { or disability }\end{array}$ & $\underline{\mathbf{2 0}}$ RR1.35 (1.09. 1.69) (B) & $\begin{array}{l}\underline{15} \mathrm{OR}=1.78(0.86-3.69)(\mathrm{B}) ; \underline{\mathbf{2 2}} \mathrm{OR}=1.6(0.9- \\
3.4)(\mathrm{B})\end{array}$ \\
\hline $\begin{array}{l}\text { Low socio- } \\
\text { educational } \\
\text { level }\end{array}$ & $\begin{array}{l}\underline{15} \mathrm{OR}=3.14(1.49-6.58) ;(\mathrm{B}) \\
\underline{\mathbf{9}} \mathrm{RR}=2.1(0.7 .4 .3)(\mathrm{B})\end{array}$ & \\
\hline $\begin{array}{l}\text { Loss of } \\
\text { autonomy }\end{array}$ & & $\underline{10} \mathrm{RR}=1.4(0.7 .2 .4)(\mathrm{B})$ \\
\hline Comorbidities & & $\underline{\mathbf{1 5}} \mathrm{OR}=1.10(0.98-1.23)(\mathrm{B})$ \\
\hline $\begin{array}{l}\text { History of } \\
\text { stroke }\end{array}$ & & $\underline{20} \mathrm{RR}=1.39(0.74 .2 .58)(\mathrm{B})$ \\
\hline $\begin{array}{l}\text { History of } \\
\text { hemodialysis }\end{array}$ & & $\underline{20} \mathrm{RR}=1.54(0.86 .2 .75)(\mathrm{B})$ \\
\hline $\begin{array}{l}\text { History of } \\
\text { diabetes }\end{array}$ & & $\underline{20} R R=1.06(0.86 .1 .30)(B)$ \\
\hline $\begin{array}{l}\text { Psychiatric } \\
\text { disorders }\end{array}$ & $\begin{array}{l}\underline{10} \mathrm{RR}=2.0(1.1 .3 .6)(\mathrm{B}) ; \\
\underline{\mathbf{2}} \mathrm{OR}=2.4(1.1-5.2)(\mathrm{B}) \\
\underline{\mathbf{9}} \mathrm{RR}=3.9(1.5 .6 .5)(\mathrm{B})\end{array}$ & \\
\hline Smoking & & $\underline{22} \mathrm{OR}=1.6(0.8-3.5)(\mathrm{B})$ \\
\hline Alcohol abuse & & $\underline{22} \mathrm{OR}=0.7(0.3-1.5)(\mathrm{B})$ \\
\hline Drug abuse & $\underline{\mathbf{2 0}} \mathrm{RR}=1.39 \quad(1.09 .1 .77)(\mathrm{B})$ & $\underline{\mathbf{2 2}} \mathrm{OR}=1.7(0.9-3.5)(\mathrm{B})$ \\
\hline $\begin{array}{l}\text { Surgical ICU } \\
\text { (vs. medical) }\end{array}$ & $\underline{10} \mathrm{RR}=2.2(1.1 .4 .2)(\mathrm{B})$ & $\begin{array}{l}\underline{\mathbf{1 5}} \mathrm{OR}=1.53(0.72-3.23)(\mathrm{B}) ; \underline{\mathbf{2 0}} \mathrm{RR}=0.99 \mathrm{IC} \\
95 \%(0.82 .1 .20)(\mathrm{B})\end{array}$ \\
\hline APACHE score & $\begin{array}{l}\underline{20} \mathrm{RR}=0.90 \\
\quad(0.82 \\
0.99)(\mathrm{B})\end{array}$ & $\begin{array}{l}\underline{22} \mathrm{OR}=0.6(0.3-1.1)(\mathrm{B}) ; \underline{21} \beta=0.044(-0.051 \\
0.14)(\mathrm{B})\end{array}$ \\
\hline SOFA score & $\underline{10} \mathrm{RR}=2.1(1.2 .3 .5)(\mathrm{B})$ & $\begin{array}{l}\underline{15} \mathrm{OR}=1.28(0.70-2.36)(\mathrm{B}) ; \underline{\mathbf{2 2}} \mathrm{OR}=0.9 \\
1.7)(\mathrm{B}) ; \underline{\mathbf{2 0}} \mathrm{RR}=0.90(0.78 .1 .05)(\mathrm{B})\end{array}$ \\
\hline Sepsis & & $\begin{array}{l}\underline{22} \mathrm{OR}=1.4(0.7-2.5)(\mathrm{B}) ; \underline{20} \mathrm{RR}=0.88(0.71 . \\
1.08)(\mathrm{B})\end{array}$ \\
\hline $\mathrm{PaO} 2 / \mathrm{FiO} 2$ ratio & & $\underline{20} \mathrm{RR}=1.01(0.98 .1 .04)(\mathrm{B})$ \\
\hline $\begin{array}{l}\text { Muscular } \\
\text { blocker }\end{array}$ & & $\underline{20} \mathrm{RR}=1.00(0.87 .1 .17)(\mathrm{B})$ \\
\hline $\begin{array}{l}\text { Vasopressor } \\
\text { agents }\end{array}$ & & $\underline{20} \mathrm{RR}=0.94(0.78 .1 .14)(\mathrm{B})$ \\
\hline $\begin{array}{l}\text { Opiods (high } \\
\text { doses) }\end{array}$ & $\underline{20} \mathrm{RR}=1.11(1.03 .1 .20)(\mathrm{B})$ & $\underline{\mathbf{2 2}} \mathrm{OR}=1.1(0.6-2.0)(\mathrm{B})$ \\
\hline $\begin{array}{l}\text { Benzodiazepines } \\
\text { (high doses) }\end{array}$ & $\begin{array}{l}\underline{\mathbf{1 0}} \mathrm{RR}=2.1(1.2 .3 .5)(\mathrm{B}) \\
\underline{\mathbf{9}} \mathrm{R} R=2.4(1.1 .3 .8)(\mathrm{B})\end{array}$ & $\begin{array}{l}\underline{\mathbf{1 5}} \mathrm{OR}=1.29(0.64-2.58)(\mathrm{B} ;) \underline{\mathbf{2 2}} \mathrm{OR}=1.3(0.7- \\
2.6)(\mathrm{B})\end{array}$ \\
\hline $\begin{array}{l}\text { Corticoids (high } \\
\text { doses) }\end{array}$ & & $\begin{array}{l}\underline{\mathbf{2 2}} \mathrm{OR}=1.9(0.7-4.8)(\mathrm{B}) ; \underline{\mathbf{2 1}} \beta=-0.089(-0.23 \\
0.055)(\mathrm{B}) ; \underline{\mathbf{2 0}} \mathrm{RR}=1.02(0.96 .1 .08)(\mathrm{B})\end{array}$ \\
\hline $\begin{array}{l}\text { Administration } \\
\text { of GM-CSF }\end{array}$ & $\underline{\mathbf{2 1}} \beta=2.8 \quad(0.37 .5 .2)(\mathrm{A})$ & \\
\hline
\end{tabular}




\begin{tabular}{|c|c|c|c|}
\hline $\begin{array}{l}\text { hypoglycemia } \\
\text { (Frequent or } \\
\text { severe) }\end{array}$ & $\underline{\mathbf{9}} \mathrm{RR}=2.6(1.2 .4 .2)(\mathrm{B})$ & & $\begin{array}{l}\underline{15} \mathrm{OR}=1.42(0.74-2.70)(\mathrm{B}) ; \underline{\mathbf{2 0}} \mathrm{RR}=1.00(0.91 . \\
1.10)(\mathrm{B})\end{array}$ \\
\hline $\begin{array}{l}\text { Prolonged } \\
\text { sedation }\end{array}$ & & & $\underline{15} \mathrm{OR}=1.02(0.91-1.14)(\mathrm{B})$ \\
\hline $\begin{array}{l}\text { Renal } \\
\text { replacement } \\
\text { therapy }\end{array}$ & & & $\underline{20} \mathrm{RR}=0.69(0.45 .1 .04)(\mathrm{B})$ \\
\hline $\begin{array}{l}\text { Duration of } \\
\text { mechanical } \\
\text { ventilation }\end{array}$ & & & $\underline{20} \mathrm{RR}=1.02(0.95 .1 .08)(\mathrm{B})$ \\
\hline $\begin{array}{l}\text { ICU length of } \\
\text { stay }\end{array}$ & & & $\begin{array}{l}\underline{\mathbf{1 5}} \mathrm{OR}=0.99(0.98-1.01)(\mathrm{B}) ; \underline{\mathbf{2 2}} \mathrm{OR}=1.2(0.6-2.2) \\
(\mathrm{B}) ; \underline{\mathbf{2 0}} \mathrm{RR}=1.01 \quad(0.95 .1 .07)(\mathrm{B})\end{array}$ \\
\hline Delirium & & & $\begin{array}{l}\underline{\mathbf{1 5}}(\mathrm{OR}=1.00(0.92-1.10)(\mathrm{B}) ; \underline{\mathbf{2 2}} \text { OR } 0.7(0.4- \\
1.3)(\mathrm{B})\end{array}$ \\
\hline $\begin{array}{l}\text { Cognitive } \\
\text { disorders }\end{array}$ & & & $\underline{\mathbf{2 4}}(\mathrm{OR}=2.33(0.83-6.55)(\mathrm{B})$ \\
\hline $\begin{array}{l}\text { SF-36 (mental } \\
\text { component) }\end{array}$ & $\underline{\mathbf{2 2}} \mathrm{OR}=4.2(1.9-9.0)(\mathrm{B})$ & & - \\
\hline $\begin{array}{l}\text { SF-36 (physical } \\
\text { component) }\end{array}$ & $\underline{\mathbf{2 2}} \mathrm{OR}=2.8(1.3-6.1)(\mathrm{B})$ & & - \\
\hline $\begin{array}{l}\text { Loss of } \\
\text { autonomy } \\
\text { (IADL) }\end{array}$ & & & $\underline{15}(\mathrm{OR}=1.29(0.67-2.47)(\mathrm{B})$ \\
\hline $\begin{array}{l}\text { Return to } \\
\text { employment }\end{array}$ & & $\begin{array}{l}\underline{11} \text { OR } 0.21 ; \\
{[0.07} \\
0.62] \text { (B) }\end{array}$ & \\
\hline
\end{tabular}

ARDS Acute respiratory distress syndrome; BMI: body mass index; APACHE (Acute Physiology and Chronic Health Evaluation III); PaO2: partial arterial pressure of oxygen; FiO2: Fraction of inspired oxygen; GM-CSF: Granulocytemacrophage colony-stimulating factor; SOFA; sequential organ failure assessment; IADL: Instrumental Activities of Daily Living; ICU: Intensive care unit

Results are given as odd ratio (OR) relative risk (RR) or correlation coefficients $(\beta)$ and 95\% confidence interval (A) Randomized control trial (B) cohort study (C) case-control study 
Table 6 Factor associated with anxiety disorder in ARDS patients

\begin{tabular}{|c|c|c|c|}
\hline $\begin{array}{l}\text { Potential Patient- } \\
\text { related Risk } \\
\text { Factors } \\
\end{array}$ & Risk factors for ARDS & $\begin{array}{l}\text { Protective } \\
\text { factors }\end{array}$ & No significative association \\
\hline Young age & $\begin{array}{l}\underline{21} \beta=-0.13(-0.21,-0.053) \\
\text { (B); } 20 \text { RR } 1.16(1.07,1.26) \\
\text { (B) }\end{array}$ & & $\begin{array}{l}\underline{17} \mathrm{OR}=1.01(0.99,1.04)(\mathrm{B}) ; \underline{22} \mathrm{OR}=1.1 \\
(0.6,2.0)(\mathrm{B})\end{array}$ \\
\hline Women & $\underline{20} R R=1.43(1.18,1.74)(B)$ & & $\begin{array}{l}\underline{17} \mathrm{OR}=1.13(0.57,2.22),(\mathrm{B}) ; \underline{21} \beta=0.38 \\
(-1.8,2.6)(\mathrm{B})\end{array}$ \\
\hline High BMI & $\begin{array}{l}\underline{\mathbf{1 7}} \mathrm{OR}=1.06(1.00,1.13)(\mathrm{B}) \\
\underline{\mathbf{2 0}} \mathrm{RR}=1.14(1.03,1.26)(\mathrm{B})\end{array}$ & & \\
\hline $\begin{array}{l}\text { Socio-educational } \\
\text { level }\end{array}$ & & & $\underline{17} \mathrm{OR}=0.59(0.28,1.21)(\mathrm{B})$ \\
\hline Profession & $\begin{array}{l}\underline{\mathbf{2 0}} \mathrm{RR}=1.26(1.05,1.52) \\
\text { unemployed_(B) }\end{array}$ & & $\begin{array}{l}\underline{\mathbf{1 7}} \mathrm{OR}=0.71(0.36,1.42)(\mathrm{B}) ; \underline{\mathbf{2 2}} \mathrm{OR}=1.3 \\
(0.7,2.4)(\mathrm{B})\end{array}$ \\
\hline Comorbidities & $\underline{\mathbf{1 7}} \mathrm{OR}=1.28(1.00,1.64)(\mathrm{B})$ & & \\
\hline $\begin{array}{l}\text { History of } \\
\text { cardiovascular } \\
\text { disease }\end{array}$ & & & $\begin{array}{l}\underline{17} \mathrm{OR}=0.26(0.06,1.10)(\mathrm{B}) ; \underline{\mathbf{2 0}} \mathrm{RR}=1.04 \\
(0.49,2.18)(\mathrm{B})\end{array}$ \\
\hline $\begin{array}{l}\text { History of } \\
\text { hemodialysis }\end{array}$ & & & $\underline{20} \mathrm{RR}=1.05(0.63,1.77)(\mathrm{B})$ \\
\hline $\begin{array}{l}\text { History of } \\
\text { diabetes }\end{array}$ & & & $\underline{20} \mathrm{RR}=0.99(0.82,1.20)(\mathrm{B})$ \\
\hline $\begin{array}{l}\text { Chronic lung } \\
\text { disease }\end{array}$ & $\underline{\mathbf{1 7}} \mathrm{OR}=3.41(1.22,9.57)(\mathrm{B})$ & & \\
\hline Smoking & & & $\underline{22} \mathrm{OR}=1.5(0.8,3.1)(\mathrm{B})$ \\
\hline Alcool dependecy & $\underline{20} \mathrm{RR}=1.45(1.18,1.79)(\mathrm{B})$ & & $\underline{22} \mathrm{OR}=1.5(0.8,3.0)(\mathrm{B})$ \\
\hline Drug abuse & & & $\begin{array}{l}\underline{17} \mathrm{OR}=1.87(0.71,4.92)(\mathrm{B}) ; \underline{\mathbf{2 2}} \text { OR } 1.6 \\
(0.7,3.5)(\mathrm{B})\end{array}$ \\
\hline $\begin{array}{l}\text { Psychiatric } \\
\text { disorders }\end{array}$ & $\begin{array}{l}\underline{\mathbf{1 7}} \text { OR=3.59 }(1.25,10.30)(\mathrm{B}) ; \\
\underline{\mathbf{2 2}} \text { OR } 2.5(1.1,5.4)(\mathrm{B})\end{array}$ & & \\
\hline $\begin{array}{l}\text { Surgical ICU (vs. } \\
\text { medical) }\end{array}$ & & & $\begin{array}{l}\underline{17} \mathrm{OR}=1.75(0.67,4.59)(\mathrm{B}) ; \underline{\mathbf{2 0}} \mathrm{RR}=1.02 \\
(0.87,1.21)(\mathrm{B})\end{array}$ \\
\hline $\begin{array}{l}\text { APACHE score }(2 \\
\text { or } 3)\end{array}$ & $\underline{\mathrm{21}} \beta=0.104(0.018,0.19)(\mathrm{B})$ & & $\begin{array}{l}\underline{\mathbf{1 7}} \mathrm{OR}=1.01(0.97,1.05)(\mathrm{B}) ; \underline{\mathbf{2 2}} \mathrm{OR}=0.7 \\
(0.3,1.2)(\mathrm{B}) ; \underline{\mathbf{2 0}} \mathrm{RR}=0.95(0.88,1,02)(\mathrm{B})\end{array}$ \\
\hline SOFA score & & & $\begin{array}{l}\underline{\mathbf{1 7}} \mathrm{OR}=1.01(0.91,1.12)(\mathrm{B}) ; \underline{\mathbf{2 2}} \mathrm{OR}=0.7 \\
(0.4,1.4)(\mathrm{B}) ; \underline{\mathbf{2 0}} \mathrm{RR}=0.88(0.73,1.07)(\mathrm{B})\end{array}$ \\
\hline Sepsis & & & $\begin{array}{l}\underline{\mathbf{1 7}} \mathrm{OR}=1.05(0.97,1.14)(\mathrm{B}) ; \underline{\mathbf{2 2}} \mathrm{OR}=1.2 \\
(0.7,2.2)(\mathrm{B}) ; \underline{\mathbf{2 0}} \mathrm{RR}=0.99(0.82,1.20)(\mathrm{B})\end{array}$ \\
\hline $\mathrm{PaO} 2 / \mathrm{FiO} 2$ ratio & & & $\underline{20} \mathrm{RR}=0.99(0.97,1.01)(\mathrm{B})$ \\
\hline $\begin{array}{l}\text { Prolonged } \\
\text { muscular } \\
\text { blockade }\end{array}$ & & & $\underline{\mathbf{2 0}} \mathrm{RR}=1.12(0.99,1.27)(\mathrm{B})$ \\
\hline $\begin{array}{l}\text { Opiods (high } \\
\text { doses) }\end{array}$ & $\underline{20} \mathrm{RR}=1.08(1.01,1.15)(\mathrm{B})$ & & $\begin{array}{l}\underline{17} \mathrm{OR}=0.98(0.50,1.92)(\mathrm{B}) ; \underline{\mathbf{2 2}} \mathrm{OR}=0.8 \\
(0.4,1.4)(\mathrm{B})\end{array}$ \\
\hline $\begin{array}{l}\text { Benzodiazepines } \\
\text { (high doses) }\end{array}$ & & & $\begin{array}{l}\underline{17} \mathrm{OR}=1.01(0.97,1.05)(\mathrm{B}) ; \underline{\mathbf{2 2}} \text { OR } 0.8 \\
(0.4-1.5)(\mathrm{B})\end{array}$ \\
\hline $\begin{array}{l}\text { Corticoids (high } \\
\text { doses) }\end{array}$ & & $\begin{array}{l}\mathbf{2 1} \beta=-0.16 \\
(-0.29,-0.025) \\
(B)\end{array}$ & $\begin{array}{l}\underline{\mathbf{1 7}} \mathrm{OR}=0.99(0.95,1.03)(\mathrm{B}) ; \underline{\mathbf{2 2}} \mathrm{OR}=2.0 \\
(0.8,5.1)(\mathrm{B}) ; \underline{\mathbf{2 0}} \mathrm{RR}=1.02(0.97,1.07)(\mathrm{B})\end{array}$ \\
\hline $\begin{array}{l}\text { Administration of } \\
\text { GM-CSF }\end{array}$ & & & $\underline{21} \beta=1.97(-0.23,4.2)(\mathrm{A})$ \\
\hline
\end{tabular}


Hypoglycemia

(Frequent or

severe)

Renal

replacement

therapy

Duration of

mechanical

ventilation

ICU length of stay

Occurrence of

delirium

Cognitive

disorders

SF-36 (mental

component)

SF-36 (physical

component) $\underline{17} \mathrm{OR}=1.00(0.98,1.02)$ (B); $\underline{\mathbf{2 0}} \mathrm{RR}=0.96$

$(0.87,1.05)(\mathrm{B})$

ARDS : acute respiratory distress syndrom ; BMI : body mass index ; SF-HS : Short-Form Health Survey ; APACHE : Acute Physiology and Chronic Health Evaluation PaO2: partial arterial pressure of oxygen; FiO2: Fraction of inspired oxygen; GM-CSF: Granulocyte-macrophage colony-stimulating factor; SOFA: sequential organ failure assessment; ICU: intensive care unit

Results are given as odd ratio (OR) relative risk (RR) or correlation coefficients ( $\beta$ ) and 95\% confidence interval; (A) Randomized control trial (B) cohort study (C) case-control study 
Table 7 Factor associated with post-traumatic stress disorder in ARDS patients

\begin{tabular}{|c|c|c|c|}
\hline $\begin{array}{l}\text { Potential risk } \\
\text { factors }\end{array}$ & Risk factors for ARDS & $\begin{array}{l}\text { Protective } \\
\text { factors }\end{array}$ & No significant association \\
\hline Age & & & $\begin{array}{l}\underline{\mathbf{1 8}} \text { OR }=0.83(0.36,1.90)(>55 y o)(\mathrm{B}) ; \\
\underline{\mathbf{2 2}} \text { OR=1.9 }(0.9,3.8) \text { (B) }\end{array}$ \\
\hline Women & $\begin{array}{l}\underline{\mathbf{2 1}} \beta=11.6(3.4,19.7)(\mathrm{B}) ; \underline{\mathbf{2 0}} \mathrm{RR}=1.80 \\
(1.31,2.48)(\mathrm{B})\end{array}$ & & $\begin{array}{l}\underline{\mathbf{1 8}} \mathrm{OR}=1.14(0.68,1.92)(\mathrm{B}) \\
\underline{\mathbf{2 2}} \mathrm{OR}=1.7(0.8,3.3)(\mathrm{B})\end{array}$ \\
\hline BMI & & & $\begin{array}{l}\underline{\mathbf{1 8}} \mathrm{RR}=1,67(0.93,3.01) \\
(\mathrm{B}) ; \underline{\mathbf{2 0}} \mathrm{RR}=1.07(0.93,1.24)(\mathrm{B})\end{array}$ \\
\hline $\begin{array}{l}\text { Socio- } \\
\text { educational } \\
\text { level }\end{array}$ & & & $\underline{18} \mathrm{OR}=0.92(0.82,1.04)(\mathrm{B})$ \\
\hline Profession & $\underline{20} \mathrm{RR}=1.40(1.03,1.90)(\mathrm{B})$ & & \\
\hline Comorbidities & & & $\underline{18} \mathrm{RR}=1.11(0.6,2.06)(\mathrm{B})$ \\
\hline $\begin{array}{l}\text { History of } \\
\text { cardiovascular } \\
\text { disease }\end{array}$ & & & $\underline{20} R R=1.61(0.76,3.43)(B)$ \\
\hline $\begin{array}{l}\text { History of } \\
\text { hemodialysis }\end{array}$ & & & $\underline{\mathbf{2 0}} \mathrm{RR}=1.39(0.72,2.67)(\mathrm{B})$ \\
\hline $\begin{array}{l}\text { History of } \\
\text { diabetes }\end{array}$ & & & $\underline{20} \mathrm{RR}=0.85(0.63,1.14)(\mathrm{B})$ \\
\hline Smoking & & & $\begin{array}{l}\underline{\mathbf{1 8}} \mathrm{RR}=1.98(0.88,4.43)(\mathrm{B}) ; \underline{\mathbf{2 2}} \\
\mathrm{OR}=2.1(0.7,6.0)(\mathrm{B})\end{array}$ \\
\hline Drug abuse & & & $\begin{array}{l}\underline{\mathbf{1 8}} \mathrm{RR}=1.12(1.06,2.09)(\mathrm{B}) ; \underline{\mathbf{2 2}} \\
\mathrm{OR}=1.6(0.7,3.5)(\mathrm{B})\end{array}$ \\
\hline $\begin{array}{l}\text { Alcool } \\
\text { dependecy }\end{array}$ & $\underline{20} \mathrm{OR}=1.79(1.31,2.46)(\mathrm{B})$ & & $\begin{array}{l}\underline{\mathbf{1 8}} \mathrm{RR}=1.03(0.56,1.88)(\mathrm{B}) \\
\underline{\mathbf{2 2}} \mathrm{OR}=1.2(0.6,2.7)(\mathrm{B})\end{array}$ \\
\hline $\begin{array}{l}\text { Psychiatric } \\
\text { disorder }\end{array}$ & $\begin{array}{l}\underline{\mathbf{1 8}} \mathrm{RR}=1.96(1.06,3.64)(\mathrm{B}) ; \underline{\mathbf{2 2}} \mathrm{OR}=2.9 \\
(1.3,6.5)(\mathrm{B}) ; \underline{\mathbf{2 0}} \mathrm{OR}=1.39(1.06,1.83) \\
(\mathrm{B})\end{array}$ & & \\
\hline $\begin{array}{l}\text { Surgical ICU } \\
\text { (vs. Medical) }\end{array}$ & & & $\underline{20} \mathrm{RR}=1.00(0.77,1.28)(\mathrm{B})$ \\
\hline APACHE score & $\underline{21} \beta=0.48(0.16,0.8)(B)$ & $\begin{array}{l}20 R R=0.88 \\
(0.79,0.98) \\
(B)\end{array}$ & $\begin{array}{l}\underline{\mathbf{1 8}} \mathrm{OR}=0.99(0.96,1.03)(\mathrm{B}) ; \underline{\mathbf{2 2}} \\
\mathrm{OR}=0.6(0.3,1.3)(\mathrm{B})\end{array}$ \\
\hline SOFA score & & & $\begin{array}{l}\underline{22} \mathrm{OR}=1.7(0.8,3.4)(\mathrm{B}) ; \underline{20} \mathrm{RR}=0.99 \\
(0.81,1.21)(\mathrm{B})\end{array}$ \\
\hline Sepsis & $\underline{18} \mathrm{OR}=1.08(1.00,1.16)(\mathrm{B})$ & & $\begin{array}{l}\underline{22} \mathrm{OR}=1.1(0.6,2.2)(\mathrm{B}) ; \underline{20} \mathrm{RR}=0.91 \\
(0.68,1.21)(\mathrm{B})\end{array}$ \\
\hline $\mathrm{PaO}_{2} / \mathrm{FiO}_{2}$ ratio & $\underline{20} R R=1.01(0.98,1.04)(B)$ & & \\
\hline $\begin{array}{l}\text { Muscular } \\
\text { blocker }\end{array}$ & $\underline{20} R R=1.23(1.02,1.49)(B)$ & & \\
\hline $\begin{array}{l}\text { vasopressor } \\
\text { agents }\end{array}$ & & & $\underline{20} \mathrm{RR}=1.12(0.87,1.44)(\mathrm{B})$ \\
\hline $\begin{array}{l}\text { Opiods (high } \\
\text { doses) }\end{array}$ & $\underline{18} \mathrm{OR}=2.13 \quad(1.02,4.42)(\mathrm{B})$ & & $\underline{20} R R=1.09(0.98,1.22)(B)$ \\
\hline $\begin{array}{l}\text { Benzodiazepines } \\
\text { (high doses) }\end{array}$ & & & $\begin{array}{l}\underline{\mathbf{1 8}} \mathrm{OR}=1.63(0.85,3.13)(\mathrm{B}) \\
\underline{\mathbf{2 2}} \mathrm{OR}=0.8(0.4,1.5)(\mathrm{B})\end{array}$ \\
\hline $\begin{array}{l}\text { Corticoids (high } \\
\text { doses) }\end{array}$ & & $\begin{array}{l}\underline{18} \mathrm{OR}=0.91 \\
(0.84,0.99) \\
(\mathrm{B})\end{array}$ & $\begin{array}{l}\underline{\mathbf{2 2}} \mathrm{OR}=2.8(0.8,9.9)(\mathrm{B}) ; \underline{\mathbf{2 1}} \beta=-0.47 \\
(-0.95,0)(\mathrm{B}) ; \underline{\mathbf{2 0}} \mathrm{RR}=1.04(0.96,1.12) \\
(\mathrm{B})\end{array}$ \\
\hline Administration & & & $\underline{\mathbf{2 1}} \beta=6.9(0.5,13.4)(\mathrm{A})$ \\
\hline
\end{tabular}


Hypoglycemia $\quad \underline{\mathbf{2 0}} \mathrm{RR}=0.96(0.87,1.06)(\mathrm{B})$

(Frequent or

severe)

\begin{tabular}{|c|c|c|}
\hline \multicolumn{2}{|l|}{$\begin{array}{l}\text { Prolonged } \\
\text { sedation }\end{array}$} & $\underline{\mathbf{1 8}} \mathrm{OR}=1.02(0.90,1.16)(\mathrm{B})$ \\
\hline $\begin{array}{l}\text { Renal } \\
\text { replacement } \\
\text { therapy }\end{array}$ & & $\underline{20} R R=0.95(0.64,1.43)(B)$ \\
\hline $\begin{array}{l}\text { Duration of } \\
\text { mechanical } \\
\text { ventilation }\end{array}$ & & $\underline{20} R R=1.02(0.94,1.11)(B)$ \\
\hline $\begin{array}{l}\text { ICU length of } \\
\text { stay }\end{array}$ & $\underline{18} \mathrm{OR}=1.39(1.06,1.83)(\mathrm{B})$ & $\begin{array}{l}\underline{22} \mathrm{OR}=1.4(0.7,2.8)(\mathrm{B}) ; \underline{20} \mathrm{RR}=0.93 \\
(0.83,1.04)(\mathrm{B})\end{array}$ \\
\hline Delirium & & $\begin{array}{l}\underline{\mathbf{1 8}} \mathrm{OR}=0.79(0.29,2.15)(\mathrm{B}) ; \underline{\mathbf{2 2}} \\
\mathrm{OR}=1.2(0.6,2.5)(\mathrm{B})\end{array}$ \\
\hline $\begin{array}{l}\text { SF-36 (mental } \\
\text { component) }\end{array}$ & $\underline{22} \mathrm{OR}=3.7(1.5,9.0)(\mathrm{B})$ & \\
\hline
\end{tabular}

Table 7 Factor associated with post-traumatic stress disorder in ARDS patients

ARDS : acute respiratory distress syndrom ; BMI : body mass index ; APACHE : Acute Physiology and Chronic Health Evaluation PaO2: partial arterial pressure of oxygen; FiO2: Fraction of inspired oxygen; GM-CSF: Granulocytemacrophage colony-stimulating factor; SOFA: sequential organ failure assessment; ICU: Intensive care unit Results are given as odd ratio (OR) relative risk (RR) or correlation coefficients $(\beta)$ and 95\% confidence interval; (A) Randomized control trial (B) cohort study (C) case-control study

\section{Figures}




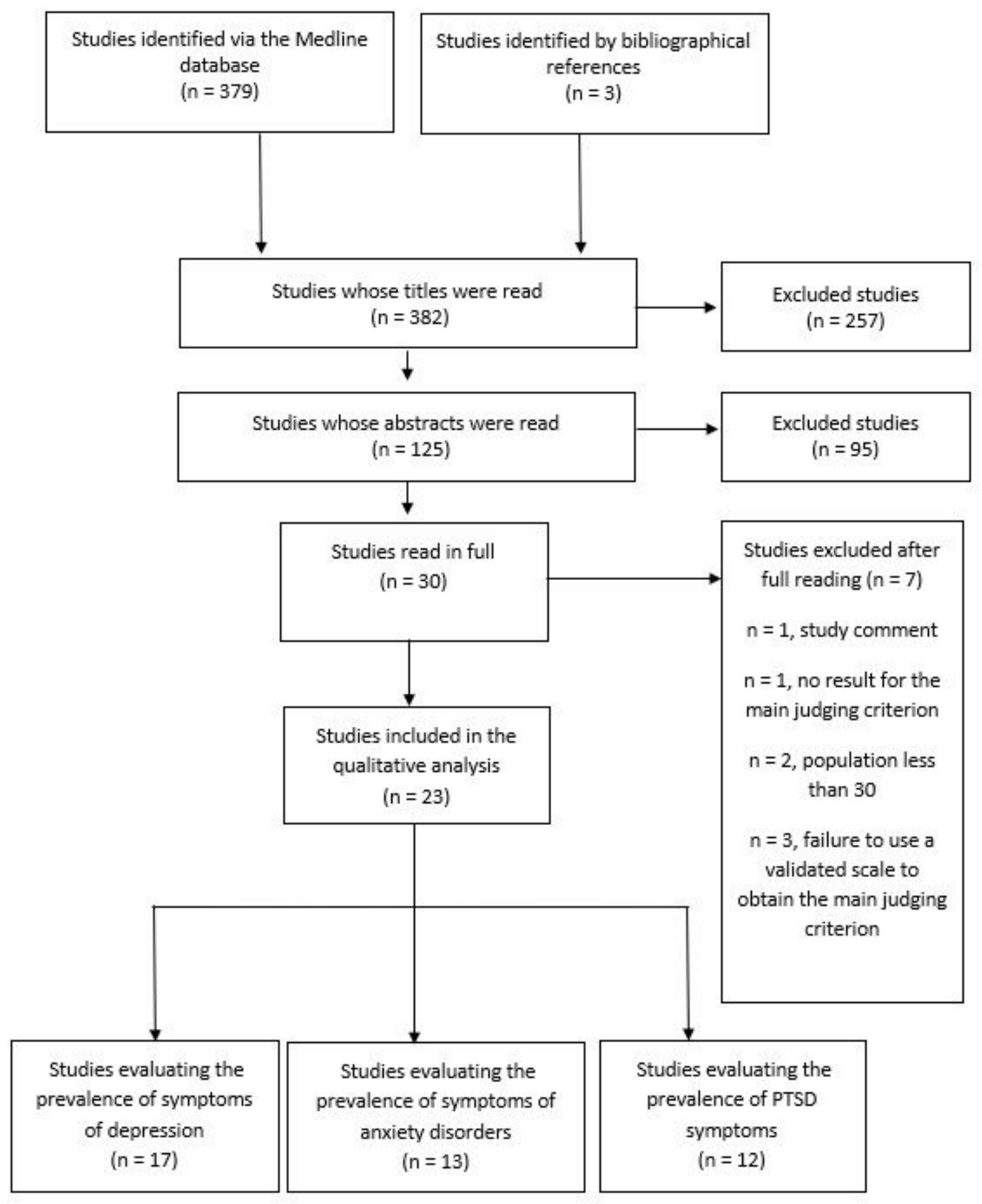

\section{Figure 1}

Flowchart showing the systematic review process 